\title{
Identifying Affective Events and the Reasons for their Polarity
}

\author{
Ellen Riloff \\ University of Utah \\ riloffecs.utah.edu
}

\section{Abstract of invited talk}

Many events have a positive or negative impact on our lives (e.g., "I bought a house" is typically good news, but "My house burned down" is bad news). Recognizing events that have affective polarity is essential for narrative text understanding, conversational dialogue, and applications such as summarization and sarcasm detection. We will discuss our recent work on identifying affective events and categorizing them based on the underlying reasons for their affective polarity. First, we will describe a weakly supervised learning method to induce a large set of affective events from a text corpus by optimizing for semantic consistency. Second, we will present models to classify affective events based on Human Need Categories, which often explain people's motivations and desires. Our best results use a co-training model that consists of event expression and event context classifiers and exploits both labeled and unlabeled texts. We will conclude with a discussion of interesting directions for future work in this area. 